Correo: innova@uide.edu.ec

\title{
La justicia redistributiva global y la complejidad de las estructuras realistas
}

\section{Global redistributive justice and the complexity of realistic structures}

\author{
¿Qué tan satisfactorio es el relato de Charles Beitz sobre la justicia redistributiva global?
}

Santiago Carranco Paredes

Universidad Internacional del Ecuador, Ecuador

Autor para correspondencia: scarrancoparedes@gmail.com

Fecha de recepción: 29 de Agosto de 2017 - Fecha de aceptación: 15 de Noviembre de 2017

\section{Resumen}

En 1973 Charles Beitz reconstruyó un modelo idealista global de justicia redistributiva utilizando los principios de la teoría Rawlsaniana, como son el velo de la ignorancia y la repartición equitativa de libertades y recursos. Beitz, que partió de premisas cosmopolitas liberales, dedicó su estudio a los desequilibrios que existen dentro del sistema internacional, planteando justamente que las causas de la desigual repartición de recursos corresponden a articulaciones sistémicas. Este texto que consta de tres apartados, discutirá si la representación de Charles Beitz sobre la justicia global es satisfactoria o no. En la primera parte se expondrá el argumento idealista del autor, en el segundo apartado se resaltarán las falacias teóricas que se puede encontrar en dicha propuesta, y la última parte se dejará para prescribir un balance de la moral realista que mantienen los estados y cómo dichos principios aún imperan dentro del sistema internacional.

Palabras Clave: justicia redistributiva; recursos naturales; desarrollo; sistema internacional

\begin{abstract}
In 1973 Charles Beitz used the principles of Rawls's Theory of Justice such as the veil of ignorance and the fair redistribution of liberties and resources to reconstruct a cosmopolitan model of redistributive justice, Beitz, who used liberal and cosmopolitan premises in his theory, has dedicated his analysis to study the imbalances that exist within the international system, rightly arguing that the causes of unfair distribution of resources are systemically produced. This text, which consists of three sections, will discuss whether Charles Beitz's representation of global justice is satisfactory or not. The first part will analyse Beitz's idealist argument, whereas the second section will expose the theoretical fallacies that can be found in Beitz's theory, while the final part will analyse the real politics among an anarchical international system.
\end{abstract}

Key words: redistributive justice; natural resources; development; international system 


\section{Introducción}

La justicia redistributiva ha sido desde tiempos inmemoriales uno de los temas más prominentes que abarca el estudio de la filosofía política. Desde la época de la antigua Grecia, autores como Epicúreo, han centrado sus estudios en los criterios de la distribución justa de recursos, sin embargo, estos se han concentrado en las desigualdades entre ciudadanos de una misma unidad estatal. No obstante, en 1973 Charles Beitz reconstruyó un modelo idealista global de justicia redistributiva utilizando los principios de la teoría Rawlsaniana, como son el velo de la ignorancia y la repartición equitativa de libertades y recursos. Beitz, que partió de premisas cosmopolitas liberales, dedicó su estudio a los desequilibrios que existen dentro del sistema internacional, planteando justamente que las causas de la desigual repartición de recursos corresponden a articulaciones sistémicas.

El autor explícitamente desafía al statu quo que impera en la política internacional e indica que el escepticismo hacia la moral realista que mantienen los estados es prominente en nuestra época. Por ende, es posible repensar una distribución equitativa de los recursos naturales de manera global.

Este texto que consta de tres apartados, discutirá si la representación de Charles Beitz sobre la justicia global es satisfactoria o no. En la primera parte se expondrá el argumento idealista del autor, en el segundo apartado se resaltarán las falacias teóricas que se pueden encontrar en dicha propuesta, y por último se prescribirá un balance de la moral realista que mantienen los estados y cómo dichos principios aún imperan dentro del sistema internacional.

\section{La justicia Redistributiva}

Beitz (1979), establece que dentro del estudio de las relaciones internacionales existen mayores preocupaciones antes que la aprehensión tradicional realista, la cual sostiene que el problema imperante en el campo de estudio de esta materia debe ser la justificación y la prevención de los conflictos armados, por lo que sus apartados implican que los arquitectos de dicha tradición y en especial los teóricos post-guerras como Morgenthau, Waltz y Mershaimer no abordan los verdaderos problemas de las relaciones mundiales (Beitz, 1979, p.3).

Para justificar su tesis, Beitz plantea que otro tipo de problemáticas deben ser las preponderantes, incluyendo temas sobre el bienestar social, la escasez de alimentos, la escasez de fuentes de energía renovable, la creciente brecha entre países ricos y pobres, la aparición de nuevos actores transnacionales y la necesidad de crear términos más equitativos en cuanto a la participación de los países en las decisiones la política transnacional y la economía mundial (Beitz, 1979, p.4-7).

Con el objetivo de erigir de una tesis alternativa que trate de manera más comprensiva el tema de la redistribución equitativa global, Beitz decidió partir desde un paradigma cosmopolita e institucionalista, el cual establece que la mejor manera de normalizar la justicia global es centrándose en principios contractuales y no utilitarios. Su argumento recae en la afirmación de que los estados ricos tienen una obligación moral con los menos desarrollados. Además, alega que las obligaciones distributivas internacionales deben basarse en principios normativos y no en valores morales volátiles maquillados en forma de cooperación internacional desinteresada. 
Beitz, a su vez rechaza la limitación doméstica que Rawls impone a su teoría de la justicia, argumentando que la globalización está transformando a la sociedad internacional en una misma unidad que puede mantener una política común; por ende, el autor resalta que los principios de Rawls pueden ser aplicados dentro de la arena global (Beitz, 1979: 127-129).

En primer lugar, cada persona debe tener el mismo derecho a un sistema de libertad para todos y, en segundo lugar, las desigualdades sociales y económicas deben arreglarse para maximizar la utilidad a los menos favorecidos y aún más importante, ofrecer a todas las personas condiciones de equitativa igualdad de libertades y de oportunidades (Rawls J., 1971, p.7).

Las disparidades tanto económicas, militares y territoriales hacen imposible la práctica de una concepción igualitaria de justicia entre las naciones. Los códigos tradicionales de justicia en las relaciones internacionales son obsoletos debido a las diferencias en términos de influencia, fruto de la riqueza y el poder inequitativo de las naciones del mundo. Partiendo de dichas premisas, se asume que un modelo de cooperación desinteresada es inconcebible y, por ende, una normativa redistributiva global podría cerrar dichas brechas de acceso a la justicia de las naciones (Beitz, 1975, págs. 362-366).

El componente más sólido del argumento de Beitz, es su visión de la distribución injusta de los recursos naturales en la tierra, en donde afirma que hay un derecho natural del pueblo sobre los recursos del planeta que va más allá de las fronteras de los países, como lo afirma Kant en la Paz Perpetua "las personas tienen derecho a la posesión comunal en la superficie de la tierra", pensamiento que, en los elementos de la política, Sidgwick lo llamó como "las utilidades derivadas de cualquier parte de la superficie de la tierra". Por otra parte, Beitz percibió a los recursos naturales de la misma forma en que Rawls observó los talentos arbitrarios innatos en las personas; sin embargo, Beitz criticó la idea redistributiva de la riqueza de Rawls basada en la premisa de que hay gente con más talento que otros, ya que analizó la complejidad de los talentos desarrollados como constitución del yo, lo que significa que una persona no necesita justificar sus talentos innatos atribuibles a la imposibilidad de disputar la genética heredada, más bien es necesario para esta persona años de práctica y sacrificio para que sus talentos puedan producir ingresos y generar riqueza.

Por otro lado, en el ámbito internacional, se espera que las zonas ricas en recursos sean explotadas con el objetivo de prosperar, lo que significa que hay lugares que debido a su falta de recursos naturales pueden alcanzar sólo un escaso nivel de bienestar, a diferencia de las zonas ricas que poseen una ventaja comparativa (Beitz, 1975, pp. 368 - 370).

\section{Limitaciones en la teoría de Beitz}

Si bien Beitz percibe el cosmopolitismo liberal como la mejor manera de lograr una redistribución equitativa de justicia en el mundo, su argumento demuestra varias falacias conceptuales y empíricas. Primero, la concepción de que el desarrollo de los países y el bienestar de sus ciudadanos son directamente proporcionales a la cantidad de recursos en su territorio es errónea, puesto que las políticas económicas y sociales integrales, así como la educación y un gobierno decente, son claramente ejemplos de factores que tienen un impacto real en la prosperidad del estado. La segunda falacia es la incompatibilidad con la teoría de la justicia de 
Rawls, la cual fue creada con el objetivo de establecer la justicia en las sociedades a nivel doméstico, contrastando así con el cosmopolitismo que busca un bienestar igualitario de los individuos en el mundo; $y$, por último, se encuentra la aplicación impracticable de su teoría en el mundo, puesto que la sistemática internacional es de carácter anárquico.

John Rawls está de acuerdo con los objetivos cosmopolitas de lograr instituciones globales decentes, garantizar los derechos humanos y satisfacer las necesidades básicas de todos, sin embargo, dichos preceptos no implica que los intentos de Beitz por llevarla al ámbito global sean capaces de cumplir los objetivos mencionados. Primero, la presunción de que la riqueza económica dependa solamente de los recursos naturales es evidentemente un argumento equivocado. Por otra parte, Rawls estableció que el estado que goza de prosperidad económica descansa sobre las virtudes políticas y civiles y no sobre la cantidad de recursos (Rawls J., 2001, p.116).

Hayward también argumentó contra Beitz, estableciendo que:

"la dotación de recursos naturales de una nación no tiene ninguna correlación particular ni con su ordenación ni con su bienestar económico. La capacidad de valorizar los recursos es el determinante clave de la riqueza, la procedencia original de los recursos es una cuestión de relativa indiferencia" (Hayward, 2006, p.353).

Hoy en día, existen varios estados que a pesar de su falta de recursos naturales se encuentran industrializados y el nivel de vida de sus ciudadanos es visiblemente superior a los países con gran cantidad de recursos. La ilustración de la situación de Japón y Venezuela es un claro ejemplo de que los recursos naturales no son una razón fundamental para que un país logre prosperidad. Venezuela es un país situado en la costa norte de América del Sur, su área es de unos $916.445 \mathrm{~km} 2$, y su población no supera los 35 millones. El territorio venezolano es rico en recursos naturales como petróleo, gas natural, mineral de hierro, oro, minerales, energía hidroeléctrica y diamantes.

Por otra parte, Japón es una isla ubicada en el Océano Pacífico en la región de Asia Oriental, su área es de $377.972 \mathrm{~km} 2$, y su población es superior a 125 millones de habitantes, lo que, comparado con Venezuela, significa menos distribución de tierra para cada uno, además, la tierra de Japón no tiene más recursos que unos pocos minerales y frutos de mar. Si el argumento de Charles Beitz es correcto, se asumiría que Venezuela debería ser un país próspero y rico, mientras que Japón debería estar luchando contra el subdesarrollo y la pobreza dada la escasez de recursos en su territorio. Sin embargo, estos supuestos son muy diferentes a la realidad, Japón es uno de los cinco países más ricos del mundo con un PIB de 4,123 billones de dólares. ${ }^{1}$ Por otra parte, el PIB de Venezuela es de 371,3 mil millones, lo que significa que el PIB japonés es once veces mayor que el venezolano.

Tabla 1: Comparación De Indicadores Macroeconómicos Entre Venezuela Y Japón

\begin{tabular}{ll}
\hline Japón & Venezuela \\
\hline
\end{tabular}

${ }^{1}$ Datos del Banco Mundial 2015. 


\begin{tabular}{lll}
\hline Superficie & $377.972 \mathrm{~km}^{2}$ & $916.445 \mathrm{~km}^{2}$ \\
& & \\
Población & 126.96 millones & 31.11 millones \\
& & \\
PIB & 4,123 billón & 371,3 mil millones \\
PIB Per & $32.477,2$ & $12.265,0$ \\
cápita & 0.891 & 0.762 \\
IDH & 0.32 & 0.46 \\
Índice de & Recursos minerales y & Petróleo, minerales, productos agrícolas, Piedras preciosas, \\
Recursos & Frutos de mar & Metales Preciosos productos pesqueros. \\
Naturales & \multicolumn{2}{c}{ Elaborada por el autor } \\
\hline & & Fuente: Banco Mundial (2017)
\end{tabular}

En la tabla 1 es posible visualizar que la concepción de Beitz sobre la relación entre la riqueza generada por un país y la cantidad de recursos naturales es falsa. El desarrollo de Japón se debe a múltiples factores como la industrialización, el uso de la tecnología moderna y la inversión en recursos humanos, además de la característica nacional del colectivismo y la sociedad vertical (Abe y Fitzgerald, 1995). En contraste, la situación de Venezuela se explica por teorías que describen cómo los recursos naturales han jugado realmente un papel importante para el subdesarrollo de algunas sociedades. Un ejemplo es la obra de W.W Norton, que estudió cómo el descubrimiento de reservas de petróleo ha sido considerado como una desgracia para el mundo árabe.

Para Hayward (2006), "La capacidad de valorizar los recursos es el determinante clave de la riqueza; la procedencia original de los recursos es una cuestión de indiferencia relativa". Su argumento levanta dudas respecto a la percepción de Beitz en lo que es un recurso natural. La distribución de los minerales terrestres y de la biota es un hecho arbitrario, en realidad, hay virtualmente muy pocos recursos disponibles en un territorio y un país solo puede ser rico en recursos naturales si en la perspectiva global estos recursos tienen una utilidad que les da precio. Esto significa que, para que el material natural pueda ser percibido como un recurso, es necesario prospectar con el objetivo de tener cierto conocimiento de qué poder hacer con dicho material (Hayward, 2006, pp. 350-352).

Según Beitz, en la distribución de los recursos habrá gente bajo un velo de ignorancia que tendrá el conocimiento sobre la disponibilidad de recursos valiosos sobre el planeta, sin embargo, no hay tal manera de conocer las tecnologías y estudios futuros que encuentren utilidades para lo que hoy en día se perciben como materiales no valorados, o incluso la comunidad científica podría encontrar nuevos minerales o biota que podría ser considerada como un valioso recurso natural. En este caso es imposible lograr una redistribución justa de los recursos debido a la incapacidad de percibir toda la imagen de los recursos naturales que tiene el planeta.

Beitz, junto con los pensadores cosmopolitas, son partidarios de la idea de un mundo interdependiente en donde es posible una ley normativa cosmopolita para ordenarla. 
Indudablemente, las telecomunicaciones, las transacciones sociales y un sistema financiero global podrían conducirnos a pensar en un mundo virtual sin fronteras, tal como Keohane y Nye (2001) lo establecieron en "Poder e Interdependencia". Sin embargo, la realidad del mundo es muy diferente. Hoy en día, hay zonas fronterizas extremadamente fortificadas que controlan fuertemente el tránsito humano de un país a otro. Por ejemplo, la barrera México-Estados Unidos y la Barrera de Cisjordania israelí son fronteras extensas y resguardadas en todo el mundo, esto debido a las denuncias de Estados Unidos e Israel sobre la inseguridad que los carteles en México y los grupos terroristas de Palestina representan para ellos. Esta es una demostración de soberanía y seguridad sobre la relación comercial y cultural interdependiente. Por otra parte, una distribución global de los recursos podría incluso ser perjudicial para la humanidad, ya que esto podría arriesgar el frágil equilibrio del sistema global; como lo estableció Morgenthau (2006, p.3) "Saber que las naciones están sujetas a la ley moral es una cosa, mientras que pretender saber con certeza lo que es bueno y malo en las relaciones entre las naciones es otra cosa". Las relaciones internacionales son un campo muy sensible en el que cualquier norma basada en la moralidad podría ser utilizada por los estados más poderosos para obtener más poder o lograr cierto interés. Es posible hacer que dos personas cooperen, pero de nuevo en el campo estatal siempre hay un estado que va a ganar y otro que va a perder (Mearsheimer, 1994 - 1995).

Para la tradición realista el sistema internacional tiene una naturaleza anárquica, no hay tal cosa capaz de regular las relaciones entre los estados, debido a que estos se consideran su propia máxima autoridad y no reconocen un poder superior sobre ellos. Para persistir, el neorrealista como Waltz, argumenta que los estados tratarán de mantener el equilibrio de poder preservando el statu quo, esto significa que los estados harán lo necesario para mantener el orden en la sociedad internacional, actuando como racional y unidades autosuficientes.

A pesar de la existencia de instituciones supranacionales, los estados necesitan preservar su autosuficiencia para sobrevivir, como establece Marsheimer (1994-1995, p.15)

"Los estados más poderosos del sistema crean y dan forma a las instituciones para que puedan mantener su participación en el poder mundial, o incluso aumentarlo". Los argumentos realistas proporcionan la sensación sobre la incompatibilidad de la teoría de Beitz con la naturaleza del mundo, el autor basó su argumento pensando en la gente como unidades individuales que viven en una sociedad de frontera abierta, pero él no recordó el papel de los estados en la redistribución de recursos.

Por último, en el caso hipotético en el que el mundo se convierta en una sociedad de fronteras abiertas que acepta una normativa global basada en principios de redistribución, Beitz no responde a varias preguntas, como por ejemplo:

$\checkmark$ ¿Cuáles serán las instituciones responsables que implementarán la redistribución de recursos?

$\checkmark \quad$ ¿Dónde va a estar ubicado ese organismo supranacional?, y

$\checkmark \quad$ ¿Cómo se va a evitar que estas instituciones sean manipuladas por las fuerzas mayores como corporaciones o países poderosos? 
Por otra parte, existe un riesgo extremo de dar a una organización supranacional el suficiente poder para crear una constitución mundial, no sólo representa una amenaza para la seguridad de los estados y para sus ciudadanos, sino también significa la renuncia de los valores culturales y el nacionalismo. Wallace Brown (2009), recapituló algunas preocupaciones contra el cosmopolitismo desde las perspectivas relativistas, uno de los principales argumentos es que los cosmopolitas como Beitz no comprenden la complejidad de la cultura y la identidad. En realidad, una teoría cosmopolita occidental-céntrica no es cosmopolita en absoluto.

Además, en cada sociedad existen subgrupos con diferentes principios de moralidad, por lo tanto, existen diversas percepciones de la justicia (Brown, 2009, págs. 129-137). Por esta razón no es fácil llamar una teoría cosmopolita de la justicia erigida desde una perspectiva de un solo hombre en absoluto.

\section{La respuesta Ralwsiana}

Como se mencionó en el apartado anterior, la relación que Beitz ha hecho entre los recursos naturales y la riqueza, el desarrollo y el bienestar de los ciudadanos de un estado es errónea. Hayward y Ralws han estado en desacuerdo con este argumento que no parece tener bases sólidas, además hay claros ejemplos de estados que han sido capaces de generar riqueza y desarrollo a pesar de la escasez de recursos entre sus territorios. En segundo lugar, Beitz mantiene un concepto erróneo de lo que es un recurso natural, al no conocer las utilidades de todos los minerales y materiales aún no descubiertos, se imposibilita la distribución de los recursos naturales.

En consecuencia, las personas que hipotéticamente estarían bajo el velo de la ignorancia, no tendrían suficiente información técnica para lograr una redistribución justa. Tercero, la dificultad de una ley normativa en un mundo realista, imposibilita luchar contra ciertas fuerzas en las relaciones internacionales.

La teoría de Beitz es un ataque directo a la soberanía de los estados, a la naturaleza anárquica del sistema internacional y a los principios tradicionales que conforman el derecho internacional. Finalmente, las teorías cosmopolitas representan un riesgo definido para las culturas pluralistas del mundo. Una premisa que parte del noroeste no abordará los requisitos de justicia para los individuos de diferentes culturas del planeta. Además, la teoría de Beitz no expone cómo se distribuirán los recursos, quién los distribuirá y cómo controlar una distribución justa y honesta.

\section{Conclusiones}

Para concluir, es necesaria la regulación de disparidades no sólo entre los países desarrollados y los no industrializados, sino también entre los ricos y pobres en recursos. El sistema capitalista que se ha erigido hoy en día, es cada vez más poderoso que la naturaleza humana, por lo que es necesario algún tipo de transformación en el derecho internacional. Beitz ha intentado satisfacer esta necesidad, sin embargo, sus argumentos presentan un número de falacias. 
Para lograr un cosmopolitismo justo, en primer lugar, los principios deben ser repensados lejos de las teorías rawlsanianas creadas para lograr la justicia en un nivel doméstico. Adicionalmente, estos tienen que ser armoniosos con la naturaleza de los estados y respetar su soberanía en lugar de intentar ir en contra de las fuerzas del sistema internacional; y, finalmente estos principios deben ser construidos desde una perspectiva global y no sólo de premisas noroccidentales.

\section{Bibliografía}

Abe, E., \& Fitzgerald, R. (1995). Japanese Economic Success: Timing, Culture, and Organisational Capability. Business History , 1-31.

Banco Mundial. (2017). Datos de libre acceso del Banco Mundial. Obtenido de Datos de libre acceso del Banco Mundial: https://datos.bancomundial.org/

Beitz, C. (1975). Justice and International Relations. Philosophy \& Public Affairs, 360-389.

Beitz, C. (1979). Political Theory and International Relations. New Jersey: Princeton University Press.

Beitz, C. (1989). Political Equaity. New Jersey: Princeton University Press.

Brown, G. W. (2009). Grounding cosmopolitanism: from Kant to the idea of a cosmopolitan constitution. Edinburgh: University of Edinburgh Press.

Dunne, T., \& Schmidt, B. (2001). Realism. En J. Bayles, \& S. Steven, The Globalization of World Politics (págs. 142-161). New York: Oxford University Press.

Hayward, T. (2006). Global Justice and the Distribution of Natural Resources. Political Studies, 349-369.

Johnston, D. (2011). The theory of Justice as Fariness. En D. Johnston, A brief history of Justice (págs. 196-199). West Sussex: Wiley-Blackwell.

Keohane, R., \& Nye, J. (2001). Power and Interdependence. New York: Longman.

Mearsheimer, J. (1994-1995). The False Promise of International Institutions. International Security. Vol. 19, No. 3, pp. 5-49.

Morgenthau, H. (2006). Politics Among the Nations. New York: McGRaw Hill.

Rawls, J. (1971). A Theory of Justice. Bristol: J.W Arrowsmith Ltd.

Rawls, J. (2001). Justice as Fairness a Restatement. Cambridge, Massachusetts: The Belknap Press of Harvard University Press. 
Rawls, J. (2001). The law of peoples. Cambridge, Masachusets: Hardvard University Press. Sterba, J. (2002). Cosmpolitan Justice. Cambridge, Massachusetts: Westview Press Books. 